\title{
Existence for Korteweg-de Vries-type equation with delay
}

Zhihong Zhao ${ }^{1 *}$, Erhua Rong ${ }^{2}$ and Xiangkui Zhao ${ }^{1}$

\author{
* Correspondence: \\ zhaozhihong01@yahoo.com.cn \\ 'Department of Mathematics, \\ University of Science and \\ Technology Beijing, Beijing 100083, \\ P. R. China \\ Full list of author information is \\ available at the end of the article
}

\begin{abstract}
The existence and uniqueness of the Korteweg-de Vries type equation with time delay are investigated. The problem is formulated as an abstract quasi-linear function differential equation. The solution is studied as a fixed point of the abstract integral equation.

AMS Subject Classification: 39A14; 35G25.
\end{abstract}

Keywords: Korteweg-de Vries type equation with delay, initial-value problem, evolution system, mild solutions, semigroup

\section{Introduction}

There has been a particular interest in the theory of Korteweg-de Vries (KdV) equation due to its significance in nonlinear dispersive wave theory. Many different real-world nonlinear physical problems are modeled by this well-known equation [1-4]. For example, this equation has many direct physical applications to solids, liquids, gases, plasma, the FPU problem, rotating flow in tube [2,5], and so on. We refer the readers to Jeffrey and Kakutani [6] and Miura [7] for more applications.

Due to physical reasons, Kato [8-10], Bona and Smith [11], Goldstein [12], Bourgain [13], Colliander et al. [14] among others, considered one-dimensional initial-value problem of KdV equation, i.e., initial-value problem posed on the entire real line has been extensively studied in the last decades.

On the other hand, it has widely been argued and accepted [15,16] that for various reasons, time delay should be taken into consideration in modeling. Zhao and $\mathrm{Xu}$ $[17,18]$ has considered solitary wave solutions of the $\mathrm{KdV}$ equation with delays, yet the initial-value problem of the delay $\mathrm{KdV}$ equation has not been dealt with. Therefore, we want to incorporate a single discrete time delay $\tau \geq 0$ into $\mathrm{KdV}$ equation and consider the delay KdV-type equation's initial-value problem.

The aim of this article is to establish the existence and uniqueness for the initialvalue problem of $\mathrm{KdV}$-type equation with time delay. We may take the form as

$$
\frac{\partial u}{\partial t}+u(x, t) \frac{\partial u}{\partial x}+\frac{\partial^{3} u}{\partial x^{3}}=g(x, u(x, t-\tau)), \quad t \geq 0, x \in \mathbb{R} .
$$

where $g$ is a linear or nonlinear scalar-valued function, $\tau$ is a positive number. In our development, the second nonlinear term and the third derivative term in (1.1) will correspond to a negative generator of strongly continuous semigroup $T_{u}(t)$ of quasi-linear 
operators on a Banach space. Accordingly, our approach will rely primarily on semigroup methods and (1.1) will be treated as an abstract quasi-linear functional differential equation in a Banach space.

The rest of this article is organized as follows. Section 2 is devoted to some preliminary discussion. We establish results on evolution system $U_{v}(t, s)$ of the operator $A(v)=$ $D^{3}+u D$ where $D=\mathrm{d} / \mathrm{d} x$, such knowledge is needed for the main results. In Section 3, we reduce the existence of delay KdV-type equation (1.1) to the local existence of mild solution of abstract integral equation. In Section 4, we will investigate the classical solution of (1.1) and some remarks.

\section{Preliminary}

Before proceeding we shall set forth some notations and terminologies that will be used throughout the article. $X$ will denote a Banach space over a real or complex field. Let $C$ $=C([-\tau, 0] ; X)$ denote the Banach space of continuous $X$-valued functions on $[-\tau, 0]$ with the supremum norm $\|\cdot\|_{C}$, where $\tau>0$. For any real numbers $a \leq b, t \in[a, b]$ and any continuous functions $u:[a-\tau, b] \rightarrow X, u_{t}$ denotes the element of $C$ given by $u_{t}(\theta)=u(t$ $+\theta)$ for $\theta \in[-\tau, 0]$. For a linear or nonlinear operator $A$ from $X$ to $X$, let $\operatorname{Dom}(A)$ denote its domain. A linear operator $A: \operatorname{Dom}(A) \subset X \rightarrow X$, the resolvent set $\rho(A)$ of $A$ is the set of all complex numbers $\lambda \in \mathbb{C}$ such that $(\lambda I-A): \operatorname{Dom}(A) \rightarrow X$ is bijective. $B(X, X)$ will denote the space of bounded linear operators from $X$ to $X$ and if $A \in B(X, X)$, then $\|A\|$ is the norm of $A$. If $A$ is linear and $\lambda \in \rho(A)$, then $R(\lambda ; A)$ is $(A-\lambda I)^{-1} \in B(X, X)$.

Definition 2.1. [16] A strongly continuous semigroup $\{T(t)\}_{t \geq 0}$ of continuous operators on $X$ is a family of continuous mappings $T(t): X \rightarrow X, t \geq 0$, satisfying

(i) $T(0) x=x$, for all $x \in X$;

(ii) $T(t+s)=T(t) T(s)$, for all $s, t \geq 0$;

(iii) For $x \in X$ fixed, $T(\cdot) x:[0, \infty) \rightarrow X$ is continuous.

The infinitesimal generator $A_{T}$ of $T(t), t \geq 0$, is the function from $X$ to $X$ defined by

$$
A_{T} x=\lim _{t \rightarrow 0^{+}} \frac{T(t) x-x}{t}
$$

with $\operatorname{Dom}\left(A_{T}\right)$ all $x$ for which this limit exists.

Definition 2.2. (Chap. 5 of [19]) A two-parameter family of bounded linear operators $U(t, s), 0 \leq s \leq t \leq T$, on $X$ is called an evolution system if the following two conditions are satisfied:

(i) $U(s, s)=I, U(t, r) U(r, s)=U(t, s)$ for $0 \leq s \leq r \leq t \leq T$.

(ii) $(t, s) \rightarrow U(t, s)$ is strong continuous for $0 \leq s \leq t \leq T$.

$G(X, M, \beta)$ denotes the set of all linear operators $A$ in $X$ such that $-A$ generates a strongly continuous semigroup $T(t)$ with $\|T(t)\| \leq M e^{\beta t}$.

For every real $s$, we introduce a Hilbert space $H^{s}(\mathbb{R})$ as follow. Let $u \in L^{2}(\mathbb{R})$ and set

$$
\|u\|_{s}=\left(\int\left(1+\xi^{2}\right)^{s}|\hat{u}(\xi)|^{2} \mathrm{~d} \xi\right)^{1 / 2}
$$


where $\hat{u}$ denotes the Fourier transform of $u, \int$ is the integral over all of $\mathbb{R}$. The linear space of functions $u \in L^{2}(\mathbb{R})$ for which $\|u\|_{s}$ is finite is a pre-Hilbert space with the scalar product

$$
(u, v)_{s}=\int\left(1+\xi^{2}\right)^{s} \hat{u}(\xi) \overline{\hat{v}}(\xi) \mathrm{d} \xi
$$

The completion of this space with respect to the norm $\|\cdot\|_{s}$ is a Hilbert space which we denote by $H^{s}(\mathbb{R})$. It is clear that $H^{0}(\mathbb{R})=L^{2}(\mathbb{R})$. Obviously for $t \geq s, H^{s}(\mathbb{R}) \supset H^{t}(\mathbb{R})$ and $\|u\|_{t} \geq\|u\|_{s}$ for $u \in H^{t}(\mathbb{R})$ (see, e.g., Chap. 8 of [19]).

Let $X=L^{2}(\mathbb{R})=H^{0}(\mathbb{R})$ and $Y=H^{s}(\mathbb{R})$ with $s \geq 3$, thus $Y \subset X$. We define an operator $A_{0}$ by $\operatorname{Dom}\left(A_{0}\right)=H^{3}(\mathbb{R})$ and $A_{0} u=D^{3} u$ for $u \in \operatorname{Dom}\left(A_{0}\right)$ where $D=d / d x$. For every $v$ $\in Y=H^{s}(\mathbb{R}), s \geq 3$, an operator $A_{1}(v)$ is defined by $\operatorname{Dom}\left(A_{1}(v)\right)=H^{1}(\mathbb{R})$ and for $u \in$ $\operatorname{Dom}\left(A_{1}(v)\right), A_{1}(v) u=v D u$. Let $A(v)=A_{0}+A_{1}(v)$, we then have:

Lemma 2.1. [19] For every $v \in Y$, the operator $A(v) \in G(X, 1, \omega)$ with $\omega \geq \omega_{0}(v)=$ $c_{0}\|v\|_{Y}$, where $c_{0}>0$ is a constant independent of $v \in Y$.

Let $B_{r}$ be the ball of radius $r>0$ in $Y$ centered at the origin. By the special form of the family $A(v) \in G(X, 1, \omega), v \in B_{r}$, we get

Lemma 2.2. [8]If $v L C([0, T] ; X)$ has values in $B_{r}$ then there exists a unique evolution system $U_{v}(t, s), 0 \leq s \leq t \leq T$, in $X$ satisfying

(E1) $\left\|U_{v}(t, s)\right\| \leq e^{\omega(t-s)}$, for $0 \leq s \leq t \leq T$,

(E2) $\left.\frac{\partial^{+}}{\partial t} U_{v}(t, s) w\right|_{t=s}=-A(v(s)) w$ for $w \in Y, 0 \leq s \leq t \leq T$,

(E3) $\frac{\partial}{\partial s} U_{v}(t, s) w=U_{v}(t, s) A(v(s)) w$ for $w \in Y, 0 \leq s \leq t \leq T$.

The operators $A(v) \in G(X, 1, \omega), v \in B_{r}$ also have the following property.

Lemma 2.3. [19] There is a constant $c$ such that for every $u, v \in C([0, T] ; X)$ with values in $B_{r}$ and every $w \in Y$, we have

$$
\left\|U_{u}(t, s) w-U_{v}(t, s) w\right\|_{X} \leq c\|w\|_{Y} \int_{s}^{t}\|u(\tau)-v(\tau)\|_{X} \mathrm{~d} \tau .
$$

\section{The existence of local mild solution}

In this section, we will consider the local existence of mild solution for the initial-value problem of the KdV-type equation with delay

$$
\left\{\begin{array}{l}
\frac{\partial u}{\partial t}(x, t)+u(x, t) \frac{\partial u}{\partial x}(x, t)+\frac{\partial^{3} u}{\partial x^{3}}(x, t)=g\left(x, u_{t}(x)\right), \quad t \geq 0, x \in \mathbb{R} \\
u(x, s)=\phi(x, s), \quad-\tau \leq s \leq 0, \quad x \in \mathbb{R},
\end{array}\right.
$$

where the initial data $\varphi: \mathbb{R} \times[-\tau, 0] \rightarrow \mathbb{R}$ are continuous, $u_{t}(x) \in C([-\tau, 0] ; \mathbb{R})$, i.e., $u_{t}$ $(x)(s)=u(x, t+s),-\tau \leq s \leq 0$. We will also assume $g$ is globally Lipschitz in $C:=C([-\tau$, $0] ; X)$ uniformly in $x \in \mathbb{R}$, that is, there exists $L>0$ such that $|g(x, \varphi)-g(x, \tilde{\varphi})| \leq L\|\varphi-\tilde{\varphi}\|_{C}$ for all $\varphi, \tilde{\varphi} \in C$.

We prove our main existence theorem in an integrated form using a method derived from the fundamental results of Pazy [19], Travis and Webb [20], and Wu [16].

Motivated by $\mathrm{Wu}[16]$, we define the mild solution as following.

Definition 3.1. Let $A(v) \in G(X, 1, \omega), v \in B_{r}$ and $U_{v}(t, s), 0 \leq s \leq t \leq T$ be the evolution system given by Lemma 2.2. For every function $v \in C([0, T] ; X)$ with values in $B_{r}$ and $\varphi \in C$, a continuous solution $u$ of the integral equation 


$$
u(t)=U_{v}(t, 0) \phi+\int_{0}^{t} U_{v}(t, r) f(r, u(r-\tau)) d r
$$

will be called a mild solution of the initial-value problem

$$
\left\{\begin{array}{l}
\frac{\partial}{\partial t} u(x, t)=A(v) u+f(t, u(x, t-\tau)), \quad 0 \leq t \leq T, x \in \mathbb{R}, \\
u(x, s)=\phi(x, s), \quad-\tau \leq s \leq 0, \quad x \in \mathbb{R}
\end{array}\right.
$$

Define $F: C \rightarrow X$ by $F(\phi)(x):=g(x, \phi(x, \cdot)), x \in \mathbb{R}$. Then $F$ is globally Lipschitz in $C$, that is, $\|F(\varphi)-F(\tilde{\varphi})\|_{X} \leq L\|\varphi-\tilde{\varphi}\|_{C}$ for $\tilde{\varphi}, \varphi \in C$. For any given $v \in C([0, T], X)$ with the value in $B_{r}$, we have

Theorem 3.1. Let $F:[0, T] \times C \rightarrow X$ be continuous and satisfy a Lipschitz condition

$$
\|F(t, \varphi)-F(t, \tilde{\varphi})\|_{X} \leq L\|\varphi-\tilde{\varphi}\|_{C}, \quad t \in[0, T], \varphi, \tilde{\varphi} \in C,
$$

where $L$ is a positive constant. Then for a given $\varphi \in C$, there exists a unique continuous function $u:[-\tau, T] \rightarrow X$ which solves the following initial value problem of abstract integral equation

$$
\left\{\begin{array}{l}
u(t)=U_{v}(t, 0) \phi(0)+\int_{0}^{1} U_{v}(t, s) F\left(s, u_{s}\right) \mathrm{d} s, \quad 0 \leq t \leq T, \\
u_{0}=\phi .
\end{array}\right.
$$

Proof. For any given $v \in C([0, T], X)$ with the values in $B_{r}$, there exists a unique evolution system $U_{\nu}(t, s), 0 \leq s \leq t \leq T$ in $X$ satisfying $\left\|U_{\nu}(t, s)\right\| \leq e^{\omega(t-s)}$ for $t \geq 0$, where $\omega$ is a fixed constant. For any continuous function $w:[-\tau, T] \rightarrow X, U_{v}(t, s) F\left(s, w_{s}\right)$ is continuous in $s \in[0, t]$ by virtue of the continuity of $F$, the continuity of $w_{s}$ as a function in $s$ from $[0, t]$ to $C$ and the continuity of $U_{v}(t, s), 0 \leq s \leq t \leq T$. Define

$$
u^{0}(t)=\left\{\begin{array}{l}
\phi(t), \quad t \in[-\tau, 0] \\
U_{v}(t, 0) \phi(0), \quad t \in[0, T]
\end{array}\right.
$$

In general, for each positive integer $n$, define

$$
u^{n}(t)=\left\{\begin{array}{l}
\phi(t), \quad t \in[-\tau, 0] \\
U_{v}(t, 0) \phi(0)+\int_{0}^{t} U_{v}(t, s) F\left(s, u_{s}^{n-1}\right) \mathrm{d} s, \quad t \in[0, T] .
\end{array}\right.
$$

Since $F$ is continuous, there exists $N$ such that $\left\|F\left(s, u_{s}^{0}\right)\right\|_{X} \leq N$ for $0 \leq s \leq T$. Then for $0 \leq s \leq T$, we have

$$
\left\|u^{1}(t)-u^{0}(t)\right\|_{X} \leq t e^{\omega T} N,
$$

and, in general,

$$
\left\|u^{n}(t)-u^{n-1}(t)\right\|_{X} \leq N L^{n-1} e^{n \omega T} \frac{t^{n}}{n !} .
$$

Thus, for any natural number $m$ and $t \in[-\tau, T]$,

$$
\sum_{k=1}^{m}\left\|u^{k}(t)-u^{k-1}(t)\right\|_{X} \leq \sum_{k=1}^{m} \frac{N L^{k-1} e^{k \omega T} t^{k}}{k !} \leq \frac{N}{L} e^{L T e^{\omega T}} .
$$


By Weierstrass test, the function series

$$
u^{0}(t)+\left[u^{1}(t)-u^{0}(t)\right]+\cdots+\left[u^{n}(t)-u^{n-1}(t)\right]+\cdots
$$

is uniformly convergent on $[-\tau, T]$, that is, the limit $u(t):=\lim _{n \rightarrow \infty} u^{n}(t)$ exists uniformly on $[-\tau, T]$ and $u(t)$ is continuous on $[-\tau, T]$.

To establish that $u(t)$ satisfies (3.3), we observe that

$$
\begin{aligned}
& \left\|u(t)-U_{v}(t, 0) \phi(0)-\int_{0}^{t} U_{v}(t, s) F\left(s, u_{s}\right) \mathrm{d} s\right\|_{X} \\
& \leq\left\|u(t)-u^{n}(t)\right\|_{X}+\| \int_{0}^{t} U_{v}(t, s)\left[F\left(s, u_{s}\right)-F\left(s, u_{s}^{n-1}\right)\right] \quad \leq 2 N \sum_{k=n+1}^{\infty} L^{k-1} e^{k \omega T} \frac{t^{k}}{k !} .
\end{aligned}
$$

Finally, to verify the uniqueness assertion, we suppose $u^{*}(t)$ satisfies (3.3), that is,

$$
u^{*}(t)=U_{v}(t, 0) \phi(0)+\int_{0}^{t} U_{v}(t, s) F\left(s, u_{s}^{*}\right) \mathrm{d} s, \quad 0 \leq t
$$

Thus,

$$
u(t)-u^{*}(t)=\int_{0}^{t} U_{v}(t, s)\left[F\left(s, u_{s}\right)-F\left(s, u_{s}^{*}\right)\right] \mathrm{d} s .
$$

Let $K=\max _{0 \leq s \leq T}\left\|u_{s}-u_{s}^{*}\right\|_{C}$, then (3.4) yields

$$
\left\|u(t)-u^{*}(t)\right\|_{X} \leq K^{*} e^{\omega T} t, \quad K^{*}=L K \text { for } t \in[0, T] .
$$

Substituting above inequality into (3.4), we get

$$
\left\|u(t)-u^{*}(t)\right\|_{X} \leq K^{*} e^{2 \omega T} \frac{t^{2}}{2 !} \quad \text { for } t \in[0, T] .
$$

Then

$$
\left\|u(t)-u^{*}(t)\right\|_{X} \leq K^{*} e^{k \omega T} \frac{t^{k}}{k !}, k=1,2, \ldots
$$

Let $k \rightarrow \infty$, we have $\left\|u(t)-u^{*}(t)\right\|_{X}=0$. Therefore, $u(t) \equiv u^{*}(t)$ for $t \in[-\tau, T]$ and the proof is complete.

For every given function $\varphi \in C$ and $v \in C([0, T] ; X)$ with values in $B_{r}$, the initialvalue problem

$$
\left\{\begin{array}{l}
\frac{d u}{d t}=A(v) u+F, \quad \text { for } 0 \leq t \leq T \\
u_{0}=\phi
\end{array}\right.
$$

where $A(v)=v D+D^{3}$, possesses a unique mild solution.

We note that (E2) and (E3) of Lemma 2.2 imply the following conditions, respectively (Chap. 5 of [19]): 
(E4) For every $u \in C\left(\left[0, T^{0}\right]: X\right)$ where $0 \leq T^{\prime} \leq T$, satisfying $u(t) \in B_{r}$ for $0 \leq t \leq T^{\prime}$, we have

$$
U_{u}(t, s) Y \subset Y, \quad \text { for } 0 \leq s \leq t \leq T^{\prime}
$$

and $U_{u}(t, s)$ is strongly continuous in $Y$ for $0 \leq s \leq t \leq T$.

(E5) Closed convex bounded subset of $Y$ are also closed in $X$.

It is not difficult to obtain the following result.

Corollary 3.2. If $F$ with values in $Y$ satisfies the conditions of Theorem 3.1, then for every $g \in C([-\tau, T] ; Y)$ and $v \in C([0, T], X)$ with the value in $B_{r}$, the integral equation

$$
w(t)=\left\{\begin{array}{l}
g(t)+\int_{0}^{t} U_{v}(t, s) F\left(s, w_{s}\right) \mathrm{d} s, 0 \leq t \leq T, \\
g(t), \quad \tau \leq t \leq 0
\end{array}\right.
$$

has a unique solution $w \in C\left(\left[0, T^{\prime}\right] ; Y\right)$.

Let $C([-\tau, 0] ; Y)$ denote the Banach space of continuous $Y$-valued function on $[-\tau, 0]$ with supremum norm $\|\cdot\|_{C_{Y}}$. Next, we consider the following initial value problem of integral equation

$$
\left\{\begin{array}{l}
u(t)=U_{u}(t, 0) \phi(0)+\int_{0}^{t} U_{u}(t, s) F\left(s, u_{s}\right) \mathrm{d} s, \quad 0 \leq t \leq T, \\
u_{0}=\phi
\end{array}\right.
$$

Theorem 3.3. If $F:[0, T] \times C \rightarrow X$ with values in $Y$ and satisfy a Lipschitz condition

$$
\|F(t, \varphi)-F(t, \tilde{\varphi})\|_{Y} \leq L\|\varphi-\tilde{\varphi}\|_{C}, \quad t \in[0, T], \quad \varphi, \tilde{\varphi} \in C,
$$

where $L$ is a positive constant and $\phi \in C([-\tau, 0] ; Y), \phi(0) \in B \frac{r}{2}$. Then there is a $T$, $0 \leq T^{\prime} \leq T$ such that the initial-value problem of integral equation (3.5) has a unique solution $u \in C\left(\left[-\tau, T^{\circ}\right] ; X\right)$ with $u(t) \in B_{r}$ for $0 \leq t \leq T$. Moreover, the mapping $\varphi \rightarrow u$ is Lipschitz continuous from $C$ into $C\left(\left[-\tau, T^{\prime}\right] ; X\right)$.

We note that (3.6) implies (3.2). Therefore, the initial value problem (3.3) possesses a unique solution $u \in C([0, T] ; X)$. That is for every given $v \in C([0, T], X)$ with the values in $B_{r}$, (3.3) defines a mapping $v \rightarrow u=G(v)$, that is, $G: C([0, T] ; X) \rightarrow C([0, T]$; $X)$ such that

$$
u(t)=G v(t):=U_{v}(t, 0) \phi(0)+\int_{0}^{t} U_{v}(t, s) F\left(s, u_{s}\right) \mathrm{d} s, \quad 0 \leq t \leq T
$$

The fixed points of this mapping are defined to be solutions of (3.5).

Proof. We note first that from the construction of $U_{v}(t, s)$ and (E4) it follows that

$$
\left\|U_{v}(t, s)\right\|_{Y} \leq \gamma \quad \text { for } 0 \leq s \leq t \leq T
$$

where $v \in C([0, T], X)$ with the values in $B_{r}$. If $\|w\|_{Y}<r$ and $v \in B_{r}$ then

$$
\|A(v) u\|_{Y} \leq(1+r)\|u\|_{Y}
$$

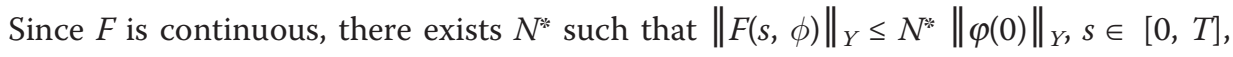


where $\varphi \in C([-\tau, 0] ; Y),\|\varphi\|_{C_{Y}} \leq \max \left\{\|\phi\|_{C_{Y}}, r\right\}$. .

Let $N:=\max \left\{L, N^{*}\right\}$, and choose

$$
T^{\prime}=\min \left\{T, \frac{r}{2 e \gamma(1+r+N)\|\phi\|_{C_{Y}}}, \frac{1}{\gamma L}, \frac{1}{N}\left(\sqrt{1+\frac{2 N}{c r}-1}\right), \frac{\ln (\omega+3 L)-\ln (3 L)}{\omega}\right\}
$$

where $c$ is the constant appearing in Lemma 2.3. We consider the subset $\Omega$ of $C([0$, $T$ ]; $X$ ) defined by

$$
\Omega=\left\{u: u \in C\left(\left[0, T^{\prime}\right] ; X\right), u(0)=\phi_{0},\left\|u(t)-\phi_{0}\right\|_{Y} \leq \frac{r}{2} \text { for } 0 \leq t \leq T^{\prime}\right\}
$$

where $\varphi_{0}:=\varphi(0)$. By our assumptions and Lemma 2.2, it is clear that $\Omega \subset B_{r}, G$ is defined on $\Omega$. From (E5) it follows that $\Omega$ is a closed subset of $C\left(\left[0, T^{0}\right] ; X\right)$. Next we consider the mapping

$$
u(t)=G v(t)=U_{v}(t, 0) \phi_{0}+\int_{0}^{t} U_{v}(t, s) F\left(s, u_{s}\right) \mathrm{d} s
$$

Clearly for any $v \in \Omega, u(0)=G v(0)=\varphi_{0}$, let $u=\varphi$ on [- $\tau$, 0]. It is clear that $G$ 's range is in $C\left(\left[0, T^{p}\right] ; X\right)$. We claim that $G: \Omega \rightarrow \Omega$. From (E4) it follows that $G(v(t)) \in$ $Y$ for $0 \leq t \leq T$, By (3.7) and (3.8), we have

$$
\begin{aligned}
& \left\|u(t)-\phi_{0}\right\|_{Y}=\left\|G v(t)-\phi_{0}\right\|_{Y} \\
\leq & \left\|U_{v}(t, 0) \phi_{0}-\phi_{0}\right\|_{Y}+\int_{0}^{t}\left\|U_{v}(t, s) F\left(s, u_{s}\right)-F(s, \phi)\right\|_{Y} \mathrm{~d} s+\int_{0}^{t}\left\|U_{v}(t, s) F(s, \phi)\right\|_{Y} \mathrm{~d} s \\
\leq & \int_{0}^{t}\left\|U_{v}(t, s) A(v) \phi_{0}\right\|_{Y} \mathrm{~d} s+\int_{0}^{t}\left\|U_{v}(t, s)\right\|_{Y}\left\|F\left(s, u_{s}\right)-F(s, \phi)\right\|_{Y} \mathrm{~d} s \\
& +\int_{0}^{t}\left\|U_{v}(t, s) F(s, \phi)\right\|_{Y} \mathrm{~d} s \\
\leq & \gamma(1+r)\left\|\phi_{0}\right\|_{Y} T^{\prime}+\gamma L \int_{0}^{t}\left\|u_{s}-\phi\right\|_{C} \mathrm{~d} s+\gamma N\left\|\phi_{0}\right\|_{Y} T^{\prime}
\end{aligned}
$$

From the last inequality it follows that

$$
\left\|u_{t}-\phi\right\|_{C_{Y}} \leq \gamma T^{\prime}(1+r+N)\|\phi\|_{C_{Y}}+\gamma L \int_{0}^{t}\left\|u_{s}-\phi\right\|_{C_{Y}} \mathrm{~d} s
$$

By Gronwall's inequality and (3.9), we get

$$
\left\|u_{t}-\phi\right\|_{C_{Y}} \leq \gamma T^{\prime}(1+r+N)\|\phi\|_{C_{Y} e^{\gamma L T^{\prime}}} \leq \frac{r}{2} .
$$

Obviously, $\left\|u(t)-\phi_{0}\right\|_{Y} \leq \frac{r}{2}$. Therefore $G: \Omega \rightarrow \Omega$. 
Moreover, if $v_{1}, v_{2} \in \Omega$ and $u_{1}=G\left(v_{1}\right), u_{2}=G\left(v_{2}\right)$, then by Lemma 2.3, we get

$$
\begin{aligned}
& \left\|u_{1}(t)-u_{2}(t)\right\|_{X} \\
\leq & \left\|U_{v 1}(t, 0) \phi_{0}-U_{v_{2}}(t, 0) \phi_{0}\right\|_{X}+\int_{0}^{t}\left\|U_{v 1}(t, s) F\left(s, u_{1 s}\right)-U_{v 2}(t, s) F\left(s, u_{2 s}\right)\right\|_{X} \mathrm{~d} s \\
\leq & c\left\|\phi_{0}\right\|_{Y} \int_{0}^{t}\left\|v_{1}-v_{2}\right\|_{X} \mathrm{~d} \tau+\int_{0}^{t}\left\|\left(U_{v 1}(t, s)-U_{v 2}(t, s)\right) F\left(s, u_{1 s}\right)\right\|_{X} \mathrm{~d} s \\
& +\int_{0}^{t}\left\|U_{v_{2}}(t, s)\left(F\left(s, u_{1 s}\right)-F\left(s, u_{2 s}\right)\right)\right\|_{X} \mathrm{~d} s \\
\leq & c\left\|\phi_{0}\right\|_{Y} T^{\prime}\left\|v_{1}-v_{2}\right\|_{\infty}+c \int_{0}^{t}\left\|F\left(s, u_{1 s}\right)\right\|_{Y} \int_{0}^{s}\left\|v_{1}(\tau)-v_{2}(\tau)\right\|_{X} \mathrm{~d} \tau \mathrm{d} s \\
& +L \int_{0}^{t}\left\|U_{v_{2}}(t, s)\right\|\left\|u_{1 s}-u_{2 s}\right\|_{C} \mathrm{~d} s \\
\leq & c T^{\prime}\left\|\phi_{0}\right\|_{Y}\left\|v_{1}-v_{2}\right\|_{\infty}+c\left\|\phi_{0}\right\|_{Y} N \frac{T^{\prime 2}}{2}\left\|v_{1}-v_{2}\right\|_{\infty}+\frac{L}{\omega}\left(e^{\omega T^{\prime}}-1\right)\left\|u_{1}-u_{2}\right\|_{\infty} \\
\leq & \frac{1}{2}\left\|v_{1}-v_{2}\right\|_{\infty}+\frac{1}{3}\left\|u_{1}-u_{2}\right\|_{\infty}
\end{aligned}
$$

where $\|\cdot\|_{\infty}$ is the usual supremum norm in $C\left(\left[0, T^{0}\right] ; X\right)$. From the last inequality, it follows readily that

$$
\left\|G\left(v_{1}\right)-G\left(v_{2}\right)\right\|_{\infty} \leq \frac{3}{4}\left\|v_{1}-v_{2}\right\|_{\infty}
$$

So $G$ is a contraction. From the contraction mapping theorem it follows that $G$ has a unique fixed point $u \in \Omega$ which is the desired solution of (3.5).

The Lipschitz continuity of the map $\varphi \rightarrow u$ is the consequence of the following argument. Let $\hat{u}$ be a solution of (3.5) on $\left[0, T^{\prime}\right]$ with the initial value $\hat{\phi}$. Then

$$
\begin{aligned}
& \|u(t)-\hat{u}(t)\|_{X} \\
& \leq\left\|U_{u}(t, 0) \phi(0)-U_{\hat{u}}(t, 0) \hat{\phi}(0)\right\|_{X}+\int_{0}^{t}\left\|U_{u}(t, s) F\left(s, u_{s}\right)-U_{\hat{u}}(t, s) F\left(s, \hat{u}_{s}\right)\right\|_{X} \mathrm{~d} s \\
& \leq\left\|U_{u}(t, 0) \phi(0)-U_{\hat{u}}(t, 0) \phi(0)\right\|_{X}+\left\|U_{\hat{u}}(t, 0) \phi(0)-U_{\hat{u}}(t, 0) \hat{\phi}(0)\right\|_{X} \\
& +\int_{0}^{t}\left\|U_{u}(t, s) F\left(s, u_{s}\right)-U_{\hat{u}}(t, s) F\left(s, u_{s}\right)\right\|_{X} \mathrm{~d} s \\
& +\int_{0}^{t}\left\|U_{\hat{u}}(t, s) F\left(s, u_{s}\right)-U_{\hat{u}}(t, s) F\left(s, \hat{u}_{s}\right)\right\|_{X} \mathrm{~d} s \\
& \leq e^{\omega t}\|\phi(0)-\hat{\phi}(0)\|_{X}+c\|\phi(0)\|_{Y} \int_{0}^{t}\|u(s)-\hat{u}(s)\|_{X} \mathrm{~d} s \\
& +L \int_{0}^{t} e^{\omega(t-s)}\left\|u_{s}-\hat{u}_{s}\right\|_{C} \mathrm{~d} s+c \int_{0}^{t}\left\|F\left(s, u_{s}\right)\right\|_{Y} \int_{0}^{s}\|u(s)-\hat{u}(s)\|_{X} \mathrm{~d} \xi \mathrm{d} s \\
& \leq e^{\omega t}\|\phi(0)-\hat{\phi}(0)\|_{X}+c\|\phi(0)\|_{Y} \int_{0}^{t}\|u(s)-\hat{u}(s)\|_{X} \mathrm{~d} s \\
& +L \int_{0}^{t} e^{\omega(t-s)}\left\|u_{s}-\hat{u}_{s}\right\|_{C} \mathrm{~d} s+c N\|\phi(0)\|_{Y} T^{\prime} \int_{0}^{t}\|u(s)-\hat{u}(s)\|_{X} \mathrm{~d} s .
\end{aligned}
$$


By the fact that $\omega>0$, for $0 \leq t \leq T$, we get

$$
\begin{aligned}
& \left\|u_{t}-\hat{u}_{t}\right\|_{C} \\
& \leq e^{\omega t}\|\phi-\hat{\phi}\|_{C}+L \int_{0}^{t} e^{\omega(t-s)}\left\|u_{s}-\hat{u}_{s}\right\|_{C}+\frac{c r}{2}\left(1+N T^{\prime}\right) \int_{0}^{t}\left\|u_{s}-\hat{u}_{s}\right\|_{C} \mathrm{~d} s \\
& \leq e^{\omega t}\|\phi-\hat{\phi}\|_{C}+L e^{\omega t} \int_{0}^{t} e^{-\omega s}\left\|u_{s}-\hat{u}_{s}\right\|_{C}+\frac{c r}{2}\left(1+N T^{\prime}\right) \int_{0}^{\omega(t-s)}\left\|u_{s}-\hat{u}_{s}\right\|_{C} \mathrm{~d} s
\end{aligned}
$$

which implies, by Gronwall's inequality, that

$$
\left\|u_{t}-\hat{u}_{t}\right\|_{C} \leq e^{\left(\omega+L+\frac{c r}{2}\left(1+N T^{\prime}\right)\right)^{t}}\|\phi-\hat{\phi}\|_{C}
$$

and therefore

$$
\|u-\hat{u}\|_{\infty} \leq e^{\left(\omega+L+\frac{c r}{2}\left(1+N T^{\prime}\right)\right)^{T^{\prime}}}\|\phi-\hat{\phi}\|_{C}
$$

which yields the Lipschitz continuity of the map $\varphi \rightarrow u$ and the proof is complete. Summarizing the above theorems, we proved the following.

Theorem 3.4. Let $F:[0, T] \times C \rightarrow X$ with values in $Y$ be continuous and satisfy

$$
\|F(t, \varphi)-F(t, \tilde{\varphi})\|_{Y} \leq L\|\varphi-\tilde{\varphi}\|_{C^{\prime}} \quad t \in[0, T], \quad \varphi, \tilde{\varphi} \in C,
$$

where $L$ is a positive constant. If $\phi \in C([-\tau, 0] ; Y), \phi(0) \in B \frac{r}{2}$, then there is a $T$, 0 $\leq T^{\prime} \leq T$ such that the initial value problem (3.1) has a unique mild solution $u \in C$ $\left(\left[-\tau, T^{0}\right] ; Y\right)$ with $u(t) \in B_{r}$ for $0 \leq t \leq T^{\prime}$. Moreover, the mapping $\varphi \rightarrow u$ is Lipschitz continuous from $C$ into $C\left(\left[0, T^{\prime}\right] ; X\right)$.

\section{The classical solution}

In this section, we give the result on existence of local classical solution for (3.1), moreover, some remarks about the extension.

From (E4), it follows that for any $v \in Y, U_{u}(t, s) v$ is continuous in $Y$ for $0 \leq s \leq t \leq$ $T$. We have by (E2) of Lemma 2.2 that

$$
\begin{aligned}
\frac{\partial^{+}}{\partial t} U_{u}(t, s) v & =\lim _{h \rightarrow 0} \frac{U_{u}(t+h, s) v-U_{u}(t, s) v}{h} \\
& =\lim _{h \rightarrow 0} \frac{U_{u}(t+h, t)-I}{h} U_{u}(t, s) v=-A(u) U_{u}(t, s) v .
\end{aligned}
$$

The right-hand side of the last equality is continuous in $X$ since $t \rightarrow U_{u}(t, s) v$ is continuous in the $Y$-norm. Therefore, the right-derivative of $U_{u}(t, s) v$ is continuous in $X$ and as a consequence $U_{u}(t, s) v$ is continuously differentiable in $X$ and

$$
\frac{\partial}{\partial t} U_{u}(t, s) v=-A(u) U_{u}(t, s) v \text { for } s \leq t \leq T^{\prime} .
$$

Theorem 4.1. Assume that $F:[0, T] \times C \rightarrow Y$ is continuously differentiable, then for every $\varphi \in C, \varphi(\theta) \in B$ for $\theta \in[-\tau, 0]$ such that $\dot{\phi} \in C$ and $\dot{\phi}^{-}+A(u) \phi(0)=F$, the initial value problem (3.1) possesses a unique classical solution $u$ given by (3.5) and $u$ 
$\in C\left(\left[0, T^{\prime}\right] ; Y\right) \cap C^{1}\left(\left[0, T^{\circ}\right], X\right)$. Moreover, the mapping $\varphi \rightarrow u$ is Lipschitz continuous from $C$ into $C^{1}\left(\left[0, T^{\circ}\right] ; X\right)$.

Proof. We note first that the continuous differentiability of $F$ from $[0, T] \times C$ into $Y$ implies that $F$ is continuous in $t$ and Lipschitz continuous in $\phi$, uniformly in $t$ on [0, $T]$. Therefore, the initial-value problem (3.1) possesses a unique mild solution $u$ on [0, $T^{\circ}$ ] by Theorem 3.4. Next we show that this mild solution is continuously differentiable on $\left[0, T^{\circ}\right]$. To this end we set $B(s)=\frac{\partial}{\partial \varphi} F(s, \varphi)$ and

$$
g(t)=\left\{\begin{array}{l}
U_{u}(t, 0) F\left(0, \phi_{0}\right)-A(u) U_{u}(t, 0) \phi_{0}+\int_{0}^{t} U_{u}(t, s) \frac{\partial}{\partial s} F\left(s, u_{s}\right) \mathrm{d} s, \quad 0 \leq t \leq T^{\prime} \\
\dot{\phi}, \quad-\tau \leq t \leq 0 .
\end{array}\right.
$$

From our assumption, it follows that $g \in C([-\tau, T] ; Y)$ and that the function $h(t, \phi)=$ $B(t) \phi$ is continuous in $t$ from [0,T] into $Y$ and uniformly Lipschitz continuous in $\phi$ since $s \rightarrow B(s)$ is continuous from $\left[0, T^{0}\right]$ into $Y$. Let $w$ be the solution of the integral equation

$$
w(t)=\left\{\begin{array}{l}
g(t)+\int_{0}^{t} U_{u}(t, s) B(s) w_{s} \mathrm{~d} s, 0 \leq t \leq T^{\prime} \\
g(t), \quad-\tau \leq t \leq 0
\end{array}\right.
$$

The existence and uniqueness of $w \in C([-\tau, T] ; Y)$ follows from Corollary 3.2. Moreover, from our assumptions and the definition of $u_{s}$ we have

$$
F\left(s, u_{s+h}\right)-F\left(s, u_{s}\right)=B(s)\left(u_{s+h}-u_{s}\right)+\varepsilon_{1}(s, h)
$$

and

$$
F\left(s+h, u_{s+h}\right)-F\left(s, u_{s+h}\right)=\frac{\partial}{\partial s} F\left(s, u_{s+h}\right) \cdot h+\varepsilon_{2}(s, h)
$$

where $h^{-1}\left\|\epsilon_{i}\right\|_{X} \rightarrow 0$ as $h \rightarrow 0$ uniformly on [0, $\left.T^{\prime}\right]$ for $i=1,2$. If $w_{h}(t)=\frac{1}{h}[u(t+h)-u(t)]-w(t)$, then from the definition of $u$, (4.1), (4.2), and (4.3), we obtain

$$
\begin{aligned}
w_{h}(t)=\{ & \left.\frac{1}{h}\left[U_{u}(t+h, 0) \phi_{0}-U_{u}(t, 0) \phi_{0}\right]+A(u) U_{u}(t, 0) \phi_{0}\right\} \\
& +\frac{1}{h} \int_{0}^{t} U_{u}(t, s)\left(\varepsilon_{1}(s, h)+\varepsilon_{2}(s, h)\right) \mathrm{d} s \\
& +\int_{0}^{t} U_{u}(t, s)\left[\frac{\partial}{\partial s} F\left(s, u_{s+h}\right)-\frac{\partial}{\partial s} F\left(s, u_{s}\right)\right] \mathrm{d} s \\
& +\left[\frac{1}{h} \int_{0}^{h} U_{u}(t+h, s) F\left(s, u_{s}\right) \mathrm{d} s-U_{u}(t, 0) F\left(0, \phi_{0}\right)\right] \\
& +\int_{0}^{t} U_{u}(t, s) B(s) w_{h}(s) \mathrm{d} s .
\end{aligned}
$$


It is not difficult to see that the norm of each one of the four first terms on the right-hand side of above equation tends to zero as $h \rightarrow 0$. Therefore, we have

$$
\left\|w_{h}(t)\right\|_{X} \leq \varepsilon(h)+M * \int_{0}^{t}\left\|w_{h}(s)\right\|_{X} \mathrm{~d} s
$$

where $M^{*}=\max \left\{\left\|U_{u}(t, s)\right\|\|B(s)\|_{X}: 0 \leq s \leq t \leq T\right\}$ and $\varepsilon(h) \rightarrow 0$ as $h \rightarrow 0$. From (4.4) it follows by Gronwall's inequality that $\left\|w_{h}(t)\right\|_{X} \leq \varepsilon(h) e^{T^{\prime} M^{*}}$ and therefore $\| w_{h}$ $(t) \|_{X} \rightarrow 0$ as $h \rightarrow 0$. This implies that $u(t)$ is differentiable on [0,T] and that its derivative is $w(t)$. Since $w \in C\left(\left[-\tau, T^{\prime}\right] ; Y\right), u$ is continuously differentiable on $\left[-\tau, T^{\prime}\right]$.

Finally, we note that from the continuous differentiability of $u$ and the assumptions on the differentiability of $F$ it follows that $s \rightarrow F\left(s, u_{s}\right)$ is continuously differentiable on $[0, T]$. By [19], $u(t)$ is the classical solution of initial-value problem (3.1). The uniqueness of $u$ and the Lipschitz continuity of the map $\varphi \rightarrow u$ are obvious and the proof is complete.

Remark 4.2. The argument given above shows clearly that for some $s \geq 3$, the classical solution $u$ of initial-value problem (3.1) is obtained in the class

$$
u \in C^{1}\left(\left[0, T^{\prime}\right] ; H^{s-3}(\mathbb{R})\right) \bigcap C\left(\left[0, T^{\prime}\right] ; H^{s}(\mathbb{R})\right),
$$

where $T$ ' depends not only $\left\|\phi_{0}\right\|_{H^{3}}$, but also the function $F$. Kato [9] obtained that for $s>\frac{3}{2}, \phi \in H^{s}$, the $\mathrm{KdV}$ equation

$$
\left\{\begin{array}{l}
\frac{\partial u}{\partial t}(x, t)+u(x, t) \frac{\partial u}{\partial x}(x, t)+\frac{\partial^{3} u}{\partial x^{3}}(x, t)=0, \quad t \geq 0, x \in \mathbb{R}, \\
u(x, 0)=\phi(x) .
\end{array}\right.
$$

has a unique solution in the class (4.5). For (3.1), $s$ may be less than 3, but it is difficult to discuss.

Remark 4.3. Kato [9] also proved that a global solution exists whenever $\varphi \in H^{s}$ with $s \geq 2$ and stays in $H^{s}$. The extension of (3.1) is also difficult. We cannot discuss it like [16] since the initial-value should satisfy the condition $\|\phi(0)\|_{Y} \leq \frac{r}{2}$.

\section{Acknowledgements}

This research was partially supported by the National Nature Science Fund $(10871213,11071014,11071205)$ and by the Fundamental Research Funds for the Central Universities. The authors would like to thank the referee for their valuable comments and suggestions on the original manuscript.

\section{Author details}

${ }^{1}$ Department of Mathematics, University of Science and Technology Beijing, Beijing 100083, P. R. China ${ }^{2}$ Shanxi agricultural University, Shanxi 030801, P. R. China

\section{Authors' contributions}

ZZ carried out the local existence of mild solution of abstract integral equation and drafted the manuscript. ER carried out the existence of classical solution. XZ participated in the design of the study and helped to draft the manuscript. All authors read and approved the final manuscript.

Competing interests

The authors declare that they have no competing interests.

Received: 28 December 2011 Accepted: 21 May 2012 Published: 21 May 2012

References

1. Davidson, RC: Methods in Nonlinear Plasma Theory. Academic Press, New York (1972) 
2. Gardner, CS, Greene, JM, Kruskal, MD: Method for solving the Korteweg-de vries equation. Phys Rev Lett. 19, 1095-1097 (1967). doi:10.1103/PhysRevLett.19.1095

3. Kortweg, DJ, De Vries, G: On the change of form of long waves advancing in a rectangular canal, and on a new type of long stationary waves. Philos Mag. 5(39), 422-443 (1895)

4. Ott, E, Sudan, RN: Damping of solitary waves. Phys Fluids. 13, 1432-1434 (1970). doi:10.1063/1.1693097

5. Fermi, A, Pasta, J, Ulam, S: Studies of nonlinear problems. 1955 (1940) I. Los Alamos Report LA

6. Jeffrey, A, Kakutani, T: Weak nonlinear dispersive waves: a discussion centred around the Korteweg-de Vries equation. SIAM Rev. 14(4), 582-643 (1972). doi:10.1137/1014101

7. Miura, RM: The Korteweg-de Vries equation: a survey of results. SIAM Rev. 18(3), 412-459 (1976). doi:10.1137/1018076

8. Kato, T: Quasi-linear equations of evolution, with applications to partial differential equations. In Lecture Notes in Math, vol. 448, pp. 25-70.Springer, New York (1975). doi:10.1007/BFb0067080

9. Kato, T: On the Korteweg-de Vries equaion. Manuscripta Math. 28, 89-99 (1979). do:10.1007/BF01647967

10. Kato, T: On the Cauchy problem for the (generalized) Korteweg-de Vries equation. Stud Appl Math. 8, 93-128 (1983)

11. Bona, JL, Smith, R: The initial-value problem for the Korteweg-de Vries equation. Philos Trans R Soc Lond Ser A Math Phys Eng Sci. 278(1287), 555-604 (1975). doi:10.1098/rsta.1975.0035

12. Goldstein, JA: The KdV equation via semigroups. Lecture Notes in Pure and Appl Math. 178, 107-114 (1996)

13. Bourgain, J: Fourier transform restriction phenomena for certain lattice subsets and applications to non-linear evolution equations, Part II: the KdV equation. Geom Funct Anal. 3(3), 209-262 (1993). doi:10.1007/BF01895688

14. Colliander, J, Staffiliani, M, Staffiliani, G, Takaoka, H, Tao, T: Sharp global well-posedness for KdV and modified KdV on $\nabla$ and [001]. J Am Math Soc 16(3), 705-749 (2003). (electronic version). doi:10.1090/50894-0347-03-00421-1

15. Hale, JK, Verduyn Lunel, SM: Introduction to Functional Differential Equations. In Applied Mathematical Sciences, vol. 99, Springer, New York (1993)

16. Wu, JH: Theory and Applications of Partial Functional Differential Equations. In Applied Mathematical Sciences, vol. 119, Springe-Verlag, New York (1996)

17. Zhao, ZH: Solitary waves of the generalized KdV equation with distributed delays. J Math Anal Appl. 344, 32-41 (2008). doi:10.1016/j.jmaa.2008.02.036

18. Zhao, ZH, Xu, YT: Solitary waves for KortewegCde Vries equation with small delay. J Math Anal Appl. 368, 43-53 (2010). doi:10.1016/j.jmaa.2010.02.014

19. Pazy, A: Semigroups of linear operators and applications to partial differential equations. In Applied Mathematical Science, vol. 44,Springer-Verlag, New York (2006)

20. Travis, CC, Webb, GF: Existence and stability for partial functional differential equations. Trans Am Math Soc. 200, 395-418 (1974)

doi:10.1186/1687-1847-2012-64

Cite this article as: Zhao et al.: Existence for Korteweg-de Vries-type equation with delay. Advances in Difference Equations 2012 2012:64.

\section{Submit your manuscript to a SpringerOpen ${ }^{\odot}$ journal and benefit from:}

Convenient online submission

- Rigorous peer review

- Immediate publication on acceptance

- Open access: articles freely available online

- High visibility within the field

- Retaining the copyright to your article

Submit your next manuscript at $\boldsymbol{\wedge}$ springeropen.com 